\title{
AVALIAÇÃO DO ESTADO NUTRICIONAL EM PACIENTES COM CIRROSE HEPÁTICA
}

\author{
Assessment of nutritional status in patients with liver cirrhosis
}

\author{
Thays Santana Guerra ${ }^{1}$, Nelci Fenalt Hoehr', Ilka de Fátima Santana Ferreira Boin ${ }^{3}$
}

\section{RESUMO}

Objetivo: Avaliar o estado nutricional de pacientes com cirrose hepática e correlacionar com a gravidade da doença e parâmetros bioquímicos. Métodos: Foram estudados 32 pacientes, 12 portadores de $\mathrm{CH}$ por hepatite $\mathrm{C}$ (37,5\%), 11 por álcool (34,37\%) e nove por hepatite C + álcool (28,12\%), classificados como Child-Pugh A (38\%), B (56\%) e C (6\%) com MELD $18 \pm 5,48$. O estado nutricional foi avaliado através do IMC, pelo escore de Mendenhall e classificado segundo Blackburn. Resultados: Foi identificada desnutrição em 59,37\% dos pacientes (leve em 40,62\%; moderada em 15,62 e grave em 3,13\%) e 37,5\% com excesso de peso. O Teste t-Student não mostrou associação entre o estado nutricional e a gravidade da doença, avaliados através do Child-Pugh e MELD. Conclusão: Os resultados mostraram que a desnutrição está presente nos pacientes estudados, porém um novo cenário com aumento na prevalência de sobrepeso foi verificado independente do grau da disfunção hepática, segundo a classificação de Child-Pugh e MELD.

Descritores: Estado Nutricional; Cirrose Hepática; Transplante Hepático.

\section{Instituição:}

${ }^{1}$ Departamento de Transplante de Fígado - Gastrocentro da Universidade Estadual de Campinas. UNICAMP, Campinas/SP

${ }^{2}$ Departamento de Patologia Clínica da Universidade Estadual de Campinas - UNICAMP, Campinas/SP

${ }^{3}$ Departamento de Transplante de Fígado - Gastrocentro da Universidade Estadual de Campinas, UNICAMP, Campinas/SP

\section{Correspondência:}

Thays Santana Guerra

Rua Darcy de Oliveira, 980, Jardim Florence, CEP 13059-062, Campinas/SP

Tel: (19) 99135-1160

E-mail: thays_nutri@yahoo.com.br
Aceito em: 30/09/2013

\section{INTRODUÇÃO}

A Cirrose hepática $(\mathrm{CH})$ representa o estágio final de diversas doenças hepáticas, ${ }^{1}$ sendo caracterizada pela desorganização da arquitetura lobular do fígado, definida histologicamente por fibrose e formação de nódulos regenerativos. A $\mathrm{CH}$ é classificada conforme suas características morfológicas e suas manifestações clínicas. ${ }^{2}$

As principais causas de $\mathrm{CH}$ são as hepatites virais (B e C) e as doenças alcoólicas do fígado. A história natural da $\mathrm{CH}$ é tipicamente caracterizada por uma fase assintomática prolongada da doença compensada, apresentando tempo médio de diagnóstico de 10 a 12 anos. Durante esse período, o paciente deve ser monitorado pelo desenvolvimento de complicações ${ }^{1}$ manifestadas, como insuficiência hepática e/ou hipertensão portal, apresentando ou não associação à disfunção circulatória, revelando-se clinicamente por ascite, edema de membros inferiores, encefalopatia hepática, infecções e/ou hemorragia digestiva varicosa. ${ }^{2}$ 
A descompensação desenvolve-se quando a progressão da doença resulta em piora da hipertensão portal e diminuição da função hepática. A taxa de descompensação é aproximadamente de $5 \%$ a $7 \%$ ao ano. ${ }^{3}$ Conforme dados de uma pesquisa realizada em 2010 pelo Sistema de Informação sobre Mortalidade (SIM) ${ }^{4} \mathrm{com}$ homens de 50 a 55 anos, a cirrose hepática e outras doenças crônicas do fígado ocuparam a terceira posição no Brasil com taxa específica de mortalidade de aproximadamente 60 óbitos/100 mil habitantes.

O diagnóstico das complicações da $\mathrm{CH}$ e o seu estadiamento, de acordo com os critérios clínicos e laboratoriais de gravidade da doença, são fundamentais para o tratamento terapêutico dos portadores de hepatopatia crônica em fase cirrótica. O Modelo para Doença Hepática Terminal (Model for End-Stage Liver Disease ou MELD) ${ }^{5}$ é uma das escalas prognósticas empregadas para selecionar e priorizar os pacientes para transplante de fígado e estimar a probabilidade de morte dentro de um intervalo de tempo, além de representar uma estimativa quantitativa da reserva da função hepática e da capacidade de suportar um procedimento cirúrgico ou uma intervenção terapêutica mais agressiva. ${ }^{6}$

Para o cálculo do escore MELD, utilizam-se três parâmetros laboratoriais (creatinina, bilirrubina sérica, e Relação Normatizada Internacional da atividade da protrombina -RNI) e classificam-se os pacientes conforme a severidade da doença, em uma escala contínua e objetiva. ${ }^{7} \mathrm{O}$ acesso prioritário ao transplante é dado ao paciente com maior escore do MELD. ${ }^{8}$

Diante da gravidade das doenças hepáticas, alcoólicas ou não, um dos fatores que tem relevante importância é a desnutrição proteico-calórica que os pacientes podem apresentar, causando complicações em ascites, varizes esofágicas e encefalopatias hepáticas ${ }^{9,10}$ com impactos diretos no aumento da morbidade e mortalidade. ${ }^{7}$ A primeira causa da desnutrição na falência hepática é o déficit na alimentação, uma vez que os pacientes com doença hepática crônica normalmente apresentam náusea, anorexia e alteração no paladar, o que pode ocasionar a diminuição na ingestão alimentar. ${ }^{11}$ Em estudo conduzido por Mccullough, ${ }^{12}$ foi encontrado em hepatopatas, anorexia (87\%), perda de peso (60\%) e náusea $(55 \%)$.

A má absorção de nutrientes é outro importante contribuinte da desnutrição, pela impossibilidade do corpo absorver e utilizar todo o alimento ingerido ${ }^{9}$ devido à enteropatia, deficiência pancreática e biliar na doença hepática. ${ }^{13} \mathrm{~A}$ prevalência de desnutrição é de $65 \%$ a $90 \%$ nos casos de doença hepática avançada e, aproximadamente, 100\% nos candidatos a transplante hepático. ${ }^{11}$ Porém, estimativas recentes indicam que cerca de $30 \%$ dos pacientes submetidos a transplante hepático são obesos. ${ }^{14}$

Os distúrbios metabólicos decorrentes da hepatopatia, como a elevação do gasto energético, resistência insulínica e baixo quociente respiratório (indicando redução de glicose e aumento de oxigenação lipídica), podem contribuir para a desnutrição até em estágios iniciais. Pacientes hipermetabólicos que tendem a perder peso, são normalmente mais desnutridos e apresentam alta mortalidade quando comparados aos pacientes normometabólicos. ${ }^{15}$

A avaliação do estado nutricional deve ser realizada criteriosamente em todos os pacientes com doença hepática, utilizando métodos disponíveis como anamnese alimentar, antropometria, métodos bioquímicos e imunológicos, ${ }^{16}$ pois através desses pode-se determinar o diagnóstico nutricional no qual irá se basear a conduta de correção nutricional adequada. ${ }^{17}$

Ferreira et al, avaliaram 159 pacientes à espera pelo transplante hepático e a prevalência de desnutrição variou de 6,3 a $80,1 \%$, de acordo com os diferentes métodos utilizados. Nas fases iniciais da doença, a desnutrição é pouco diagnosticada, muitas vezes por não incluir na rotina do atendimento uma avaliação do estado nutricional do paciente hepatopata, ${ }^{18}$ ou até mesmo pela falta de um padrão ouro para avaliação dessa população. ${ }^{7}$

Este trabalho teve como objetivo avaliar o estado nutricional de pacientes cirróticos sem ascite e correlacionar com o escore do MELD, Child Pug e parâmetros bioquímicos, no ambulatório de transplante hepático da Universidade Estadual de Campinas (UNICAMP).

\section{MÉTODOS}

Trata-se de um estudo observacional, de caráter transversal, realizado em pacientes cirróticos do sexo masculino, maiores de 18 anos, acompanhados no ambulatório de transplante hepático da UNICAMP, no período de maio de 2011 a agosto de 2013. O protocolo da pesquisa foi submetido e aprovado pelo Comitê de Ética em Pesquisa da UNICAMP (CEP 969/2010). Para participação no estudo, os pacientes assinaram termo de consentimento livre e esclarecido.

O estado nutricional foi avaliado com base em parâmetros antropométricos Índice de massa corpórea (IMC= peso/ altura2) utilizando peso em kg e altura em metros; prega cutânea do tríceps (PCT) em mm; circunferência do braço (CB) em $\mathrm{cm}$, circunferência muscular do braço $(\mathrm{CMB}(\mathrm{cm})$ $=\mathrm{CB}(\mathrm{cm})-[0,314 \times$ PCT $(\mathrm{mm})])$, hematócrito em \% e contagem total de linfócitos em $\mathrm{mm} 3$.

Os valores de referência para os exames laboratoriais foram referenciados pelo Laboratório de Patologia Clínica do Hospital das Clínicas - Unicamp, considerando a normalidade (41 a 52\%) para hematócrito e para os linfócitos considerou-se depleção imunológica grave (DIG) uma contagem $<800 / \mathrm{mm} 3$, depleção imunológica moderada 
(DIM) 800 a 1200/mm3, depleção imunológica leve (DIL) 1200 a $2000 / \mathrm{mm} 3$ e faixa de normalidade $>2000 / \mathrm{mm} 3$.

O diagnóstico nutricional foi dado pela análise dos parâmetros em conjunto, isto é, a soma dos parâmetros nutricionais em percentual de adequação dividida pelo número de parâmetros avaliados, segundo proposição inicial de Mendenhall et al. ${ }^{19} \mathrm{e}$ também pelos valores referenciais do IMC proposto pela Organização Mundial da saúde. ${ }^{20}$

O valor, assim obtido, permitiu classificar os pacientes, segundo critérios de Blackburn et al., ${ }^{21}$ em eutróficos (100\% de adequação), levemente desnutridos (80-100\%), moderadamente desnutridos (60\%-80\%) e gravemente desnutridos $(<60 \%)$. A gravidade da doença hepática foi analisada pelo escore do MELD (Model for End-Stage Liver Disease) ${ }^{22}$ e segundo a classificação de Child Turcotte Pugh (CTP). ${ }^{7}$

Em relação às complicações das doenças hepáticas, foi analisada a presença de ascite e/ou edema (no momento da avaliação) e só foram incluídos os pacientes que não apresentavam essas complicações. A sobrevida (SV) e o tempo de sobrevida (TSV) dos pacientes foram calculados de acordo com o método de Kaplan - Meier, considerando a data da avaliação como inicial e a data final na conclusão do estudo estratificado em óbito ou vivo.

A análise estatística foi realizada no Statistica 11.0, Ohio, USA; usou-se teste de comparação de médias, Teste t-Student ou Mann-Whitney e, quando necessária, analise descritiva entre os grupos classificados de acordo com o estado nutricional, G (gravemente desnutrido), $M$ (moderadamente desnutrido), L (levemente desnutrido), $\mathrm{N}$ (nutrido) e $\mathrm{S}$ (sobrepeso). Os resultados foram considerados significativos quando $\mathrm{p}<0,05$.

\section{RESULTADOS}

Foram avaliados 32 pacientes do sexo masculino com diagnóstico de cirrose hepática, com idade média de 53,09 \pm 8 anos, 12 portadores de $\mathrm{CH}$ por hepatite $\mathrm{C}(37,5 \%), 11$ por álcool $(34,37 \%)$ e nove por hepatite C + álcool $(28,12 \%)$. Em relação à gravidade da doença $38 \%(n=12)$ foram classificados como Child A, 56\% Child B ( $n=18)$ e $6 \%$ Child C $(n=2)$. A média do MELD foi $18 \pm 5$. O perfil dos pacientes está descrito na Tabela 1 . A avaliação nutricional identificou que $3,13 \%$ estavam eutróficos, $40,62 \%$ apresentavam desnutrição leve, $15,62 \%$ estavam moderadamente, $3,13 \%$ gravemente desnutridos e 37,5\% com excesso de peso (Figura 1).

O estado nutricional não mostrou significância estatística quando relacionado com a gravidade da doença (MELD/ CTP), com a albumina sérica e nem com o perfil imunológico através dos linfócitos, conforme descrito na Tabela 2. Em relação à contagem total de linfócitos, $34,4 \%$ dos pacientes apresentaram DIG, 25\% DIM, 28,1\% DIL e 12,5\% linfócitos na faixa de normalidade. No período do estudo, $15,63 \%(\mathrm{~N}=5)$ dos pacientes foram transplantados; destes $20 \%$ foram a óbito $(\mathrm{N}=1) ; 84,4 \%(\mathrm{~N}=27)$ permaneceram na lista para transplante, com $9,411 \%(\mathrm{~N}=3)$ de óbitos e $89 \%$ $(\mathrm{N}=24)$ permaneceram em lista.

De acordo com o método de Kaplan - Meier, os grupos mostraram-se com sobrevida semelhante $(\mathrm{p}=0,85)$. Para a TSV entre os pacientes, quando se comparou nutrido e não nutrido, o grupo nutrido apresentou TSV de 13,1 meses versus o não nutridos de 7,04 meses, mostrando uma tendência para significância estatística $(\mathrm{p}=0,08)$

Tabela 1-Características dos pacientes

\begin{tabular}{lc}
\hline & Cirróticos N=32 \\
\hline Idade (anos) & $53 \pm 8$ \\
Vírus C & $12(37,5 \%)$ \\
\hline Álcool & $11(34,37 \%)$ \\
Vírus C +álcool & $09(28,12 \%)$ \\
CTP & \\
A & $12(38 \%)$ \\
B & $18(56 \%)$ \\
C & $02(6 \%)$ \\
MELD & $18 \pm 5$ \\
Parâmetros Antropométricos & \\
Peso (kg) & $83,27 \pm 12,43$ \\
Altura (m) & $1,72 \pm 0,08$ \\
IMC (kg/m ${ }^{2)}$ & $28,23 \pm 4,13$ \\
CB (cm) & $29,93 \pm 3,23$ \\
CMB (cm) & $25,75 \pm 2,41$ \\
PCT (mm) & $13,31 \pm 6,85$ \\
Parâmetros Bioquímicos & \\
Albumina (g/dl) & $3,31 \pm 0,44$ \\
Hematócrito (\%) & $37,48 \pm 6,40$ \\
Linfócito (mm $\left.{ }^{3}\right)$ & $1224,63 \pm 731,21$ \\
\hline
\end{tabular}

CTP-Child Turcotte Pugh; IMC-índice de massa corpórea; CB-circunferência do braço; CMB-circunferência muscular do braço; PCT-prega cutânea do tríceps.

\section{DISCUSSÃO}

A desnutrição está frequentemente associada com a doença hepática crônica, com prevalência de $20 \%$ a $80 \%$, dependendo do método de avaliação nutricional utilizado e da severidade da doença ${ }^{23-25}$ e tem sido correlacionada às taxas de morbidade e mortalidade associadas ao transplante hepático. ${ }^{26}$

A desnutrição ocorre muitas vezes por ingestão alimentar inadequada ou pela impossibilidade do organismo absorver e utilizar todo o alimento ingerido devido à enteropatia, deficiência pancreática e biliar presentes na doença hepática. 
Figura 1- Estado Nutricional dos Pacientes Cirróticos

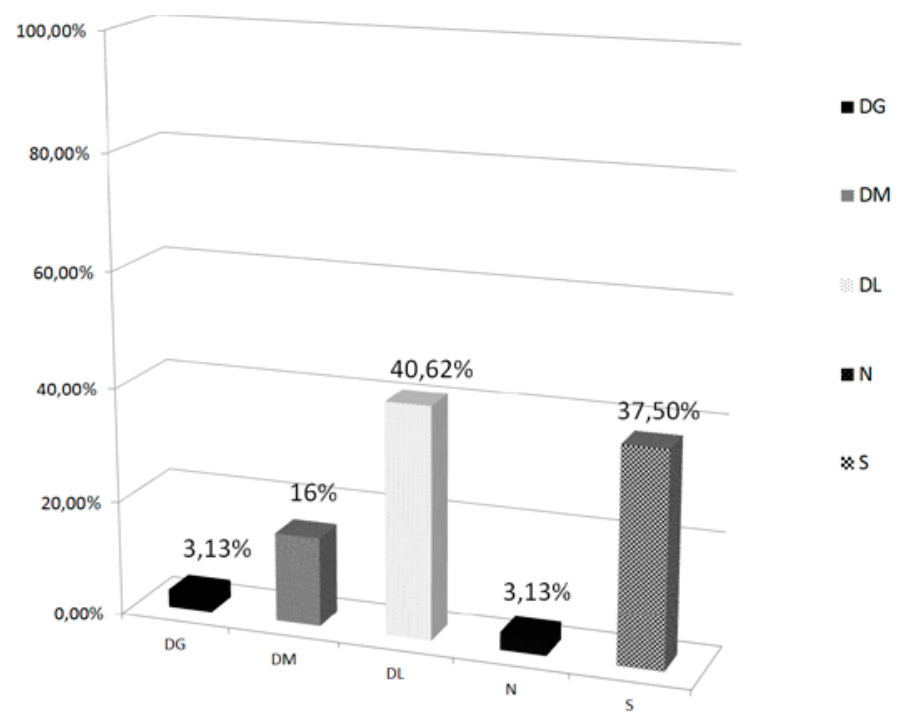

${ }^{\star} D G=$ Desnutrição grave $/{ }^{*} D M=$ Desnutrição moderada $/$

${ }^{*} \mathrm{DL}=$ Desnutrição leve $/{ }^{*} N=$ Nutrido $/{ }^{*} S=$ Sobrepeso

Leitão et al ${ }^{26}$ encontraram cerca de $2 / 3$ dos pacientes com o estado nutricional comprometido nos três estágios da classificação de Child, portanto sem relação com a gravidade da doença hepática.

Um grande estudo multicêntrico mostrou que a prevalência de desnutrição é consideravelmente maior em pacientes com comprometimento hepático mais grave $(20 \%$ a $25 \%$ em pacientes com Child A-B e > 50\% em Child C). ${ }^{27}$ Merli et al ${ }^{24}$ constataram que $56 \%$ dos pacientes pré-transplante eram desnutridos e que a presença de desnutrição não estava relacionada com a etiologia da doença hepática. No presente estudo, 59,37\% dos pacientes apresentaram um grau de desnutrição, conforme descrito na Tabela 2, sem significância estatística com a gravidade da doença.

Ferreira et al ${ }^{18}$ acompanharam 159 pacientes e a taxa de mortalidade na lista de espera para o transplante foi de $25,7 \%$ pacientes/ano e 40 pacientes foram a óbito (28\%). Neste estudo, 32 pacientes foram acompanhados e $9,4 \%$ foram a óbito pré-transplante. O grupo nutrido apresentou TSV de 13,1 meses versus o não nutridos de 7,04 meses.

Embora a desnutrição seja frequentemente observada nesses pacientes, na década passada, um novo cenário com aumento na prevalência de sobrepeso/obesidade em pacientes cirróticos tem sido apontado. A prevalência de obesidade em pacientes na lista de transplante tem se apresentado maior do que $20 \%{ }^{28}$

Zaydfudim et al, ${ }^{14}$ avaliaram 154 pacientes adultos prétransplante hepático e encontraram $41 \%$ com sobrepeso,
Tabela 2 - T-tests: Grupo Nutrido x Não Nutrido.

\begin{tabular}{lccc}
\hline & ${ }^{*}$ Grupo 1 & ${ }^{*}$ Grupo 2 & Valor $(\mathrm{p})$ \\
\hline $\begin{array}{l}\text { Idade } \\
\text { (anos) }\end{array}$ & $52,7 \pm 8,0$ & $53,615 \pm 8,4$ & $0,767204(\mathrm{NS})$ \\
MELD & $17,95 \pm 6,22$ & $18,308 \pm 3,8$ & $0,853755(\mathrm{NS})$ \\
\hline CTP pt & $7,10 \pm 1,7$ & $7,538 \pm 1,33$ & $0,446483(\mathrm{NS})$ \\
\hline $\begin{array}{l}\text { IMC } \\
\left(\mathrm{kg} / \mathrm{m}^{2}\right)\end{array}$ & $25,58 \pm 2,2$ & $32,109 \pm 4,4$ & 0,000004 \\
$\begin{array}{l}\text { PCT } \\
(\mathrm{mm})\end{array}$ & $8,0 \pm 2,21$ & $21,077 \pm 6,3$ & 0,000000 \\
$\begin{array}{l}\text { Linfócitos } \\
(\mathrm{mm})^{3}\end{array}$ & $1272,79 \pm 825,9$ & $1154,231 \pm 559,8$ & $0,655620(\mathrm{NS})$ \\
$\begin{array}{l}\text { TSV } \\
(\mathrm{meses})\end{array}$ & $13,147 \pm 10,8$ & $7,041 \pm 7,21$ & $0,085181(\mathrm{NS})$ \\
$\begin{array}{l}\text { Albumina } \\
(\mathrm{g} / \mathrm{dl})\end{array}$ & $3,384 \pm 0,5$ & $3,208 \pm 0,33$ & $0,274952(\mathrm{NS})$ \\
\hline
\end{tabular}

${ }^{*}$ Grupo 1 = Nutrido ${ }^{* *}$ Grupo 2 = Não Nutrido; IMC-índice de massa corpórea; PCT-prega cutânea do tríceps; TSV - taxa de sobrevida.

${ }^{*}$ Teste T-Student

$28 \%$ de obesos e concluíram que o excesso de peso corporal pré-transplante interfere negativamente na taxa de melhora da qualidade de vida física durante o primeiro ano após o transplante. Em três épocas diferentes de transplante hepático, a obesidade e o excesso de peso muito grave foram preditores significativos de morte, sendo que o transplante de fígado tem maior risco para os pacientes nos extremos de IMC. ${ }^{29}$ No presente estudo, encontramos $37,5 \%$ dos pacientes cirróticos com excesso de peso, o que está de acordo com os demais estudos.

Muitas anormalidades imunes têm sido associadas com a $\mathrm{CH}$, e mecanismos imunológicos podem influenciar o curso da doença por aumentar a susceptibilidade a infecções bacterianas, desarranjos hemodinâmicos e sepse. Alteração do número e função de linfócitos têm sido relatados na doença hepática. ${ }^{30}$ Neste estudo, $87,5 \%$ dos pacientes apresentaram um grau de linfocitopenia e, destes, 34,4\% de depleção imunológica grave, porém esses dados não foram estatisticamente significativos quando relacionados com o estado nutricional dos pacientes.

\section{CONCLUSÃO}

O estado nutricional dos pacientes cirróticos não teve associação com a gravidade da doença hepática (CTP e MELD) e nem com parâmetros bioquímicos.

Outros índices devem ser incluídos na avaliação desses pacientes para melhor diagnosticar o perfil nutricional. 


\section{ABSTRACT}

Purpose: To assess the nutritional status of patients with liver cirrhosis and to correlate with the severity of the disease and biochemical parameters. Methods: Thirty-two patients were studied, twelve patients with liver cirrhosis by hepatitis C (37.5\%), alcohol, eleven ( $34.37 \%$ ) and nine were studied by hepatitis C+alcohol (28.12\%), classified as Child - Pugh A (38\%), B (56\%) and C (6\%) with $18 \pm 5$ MELD . Nutritional status was assessed by BMI, Mendenhall score and Blackburn classification. Results: Malnutrition was found in $59.37 \%$ of patients (mild in $40.62 \%$, moderate in $15.62 \%$ and severe in $3.13 \%$ ) and $37.5 \%$ overweight. The Student t-test showed no association between nutritional status and the severity of the disease assessed by Child-Pugh and MELD. Conclusion: Results showed that malnutrition is present in the patients studied, but a new scenario with an increase in the prevalence of overweight was observed regardless the hepatic impairment degree according to Child- Pugh and MELD.

Keywords: Nutritional Status; Liver Cirrhosis; Liver Transplantation.

\section{REFERÊNCIAS}

1. Lefton HB, Rosa A, Cohen M. Diagnosis and Epidemiology of Cirrhosis. Med Clin North Am. 2009; 93(4):787-99.

2. Bittencourt PL, Farias AQ, Couto CA. Cirrose hepática. Clínica médica, volume 4: doenças do aparelho digestivo, nutrição e doenças nutricionais - Barueri, SP: Manole, 2009;369-83.

3. D’Amico G, Garcia-T G, Pagliaro L. Natural history and prognostic indicators of survival in cirrhosis: a systemic review of 118 studies. J Hepatol. 2006;44:217-31.

4. Ministério da Saúde - Mortalidade do adulto no Brasil: taxas de mortalidade segundo o sexo, as causas e as regiões, 2010. Disponível em: http/www. portalsaude.saude.gov.br

5. Waitzberg DL, Jesus RP, Silva AO, D'Albuquerque LAC, Camilo ME. Insuficiência Hepática Aguda e Crônica. Nutrição Oral, Enteral e Parenteral na Prática Clínica. $4^{\circ}$ Edição São PauloAtheneu, 2009.

6. Durand F, Valla D. Assessment of the prognosis of cirrhosis: Child-Pugh versus MELD. Journal of Hepatology. 2005;42:100-7.

7. Bernardi M, Gitto S, Biselli M. The MELD score in patients awaiting liver transplant: Strengths and weaknesses. Journal of Hepatology. 2011;54j:1297-306.

8. Bouygues V, Compagnon P, Latournerie M, Bardou-Jacquet E, Camus C, Lakehal M, Meunier B, Boudjema K. MELD-based graft allocation system fails to improve liver transplantation efficacy in a single-center intent-to-treat analysis. Clinics and Research in Hepatology and Gastroenterology. 2012;36:464-72.

9. O'Brien A, Williams R. Nutrition in end-stage liver disease: principle and practice. Gastroenterology. 2008;134:1729-40.

10. Kondrup J. Nutrition in end stage liver disease. Best practice \& research clinical gastroenterology. 2006;20:547-60.

11. Zhao VM, Ziegler TR. Nutrition Support in End-Stage Liver Disease. Crit Care Nurs Clin North Am. 2010;22(3):369-80.

12. McCullough AJ. Malnutrition in liver disease. Liver Transpl. 2000;6(1):85-96.
13. Maio R, Dichi JB, Burini RC. Consequências nutricionais das alterações metabólicas dos macronutrientes na doença hepática crônica. Arq Gastroenterol. 2000;37(1): 52-7.

14. Zaydfudim V, Feurer ID, Moore DE, Wisawatapnimit P, Wright JK, Pinson CW. the negative effect of pretransplant overweight and obesity on the rate of improvement in physical quality of life after liver transplantation. Surgery. 2009;146(2):174-80.

15. Selberg O, Bottcher J, Tusch G, et al. identification of high and low risk patients before liver transplantation. A prospective cohort of nutritional and metabolic parameters in 150 patients. Hepatology. 1997;25:652-7.

16. Gottschall CBA, Álvares-da-Silva MR, Camargo ACR, Burtett RM, Silveira TR. Avaliação nutricional de pacientes com cirrose pelo vírus da hepatite C: a aplicação da calorimetria indireta. Arq Gastroenterol. 2004;41:220-4.

17. Ritter L, Gazzola J. Avaliação nutricional no paciente cirrótico: uma abordagem objetiva, subjetiva ou multicompartimental? Arq Gastroentero. 2006;43:66-70.

18. Ferreira LG, Anastácio LR, Lima AS, Correia MITD. Predictors of mortality in patients on the waiting list for liver transplantation. Nutr Hosp. 2013;28(3):914-9.

19. Mendenhall CL, Tosch T, Weesner RE, Garcia-Pont $P$, Goldberg SJ, Kiernan T et al. VA cooperative study on alcoholic hepatitis. II: Prognostic significance of protein-calorie malnutrition. Am J Clin Nutr. 1986;43:213-8.

20. World health organization. Use and interpretation of anthropometric indicators of nutritional status. Bulletin World Health. Org. 1986;64:929-41

21. Blackburn GL, Bistrian BR, Maini BS, Schlamm HT, Smith MF. Nutritional and metabolic assessment of the hospitalized patient. JPEN J Parenter Enteral Nutr. 1977;1:11-22. 
22. Barshes NR, Hacker CS, Freeman Jr RB, Vierling JM, Goss JA. Justice, administrative law, and the transplant clinician: the ethical and legislative basis of a national policy on donor liver allocation. J Contemp Health Law Policy. 2007;23:200

23. Merli M, Giusto M, Giannelli V, Lucidi C, Riggio O. Nutritional Status and Liver Transplantation. Journal of Clinical and Experimental Hepatology. 2011;1(3):190-8.

24. Caregaro L, Alberino F, Amodio $P$, et al. Malnutrition in alcoholic and virus-related cirrhosis. Am J Clin Nutr. 1996; 63:602-9.

25. Leitão AVA, Castro CLN, Basile TM, Souza THS, Braulio VB. Avaliação da capacidade física e do estado nutricional em Candidatos ao transplante hepático. Rev Assoc Med Bras. 2003; 49(4):424-8.
26. Italian multicentric cooperative project on nutrition in liver cirrhosis. Nutritional status in cirrhosis. J Hepatol. 1994;21:317-25.

27. Leonard J, Heimbach JK, Malinchoc M, Watt K, Charlton M. The impact of obesity on long-term outcomes in liver transplant recipients-results of the NIDDK liver transplant database. Am J Transplant. 2008;8:667-72.

28. Dick AA, Spitzer AL, Seifert CF, et al. Liver transplantation at the extremes of the body mass index. Liver Transpl. 2009;15(8):968-77.

29. Lagadinou M, Solomou EE, Velissaris D, Theodorou GL, Karakatza $\mathrm{M}$, Gogos CA. Alterations in T-lymphocyte subpopulations in patients with complicated liver cirrhosis. Diagnostic Microbiology and Infectious Disease. 2013;75:348-56.

\section{AGRADECIMENTOS}

Os autores agradecem a equipe do Departamento de Transplante de Fígado da

Universidade Estadual de Campinas- UNICAMP

pelo estímulo e bom acolhimento prestados durante toda a pesquisa para elaboração do artigo. 